\title{
Supralinear reliability of cortical spiking from synchronous thalamic
} inputs

\author{
Wang Hsi-Ping*1, Jean-Marc Fellous*1,2, Don Spencer*1 and \\ Terrence Sejnowski*1
}

Address: 'Salk Institute, La Jolla, CA and University of California at San Diego, San Diego, CA, USA and 2University of Arizona, Tuscon, AZ, USA

Email: Wang Hsi-Ping* - ping@salk.edu

* Corresponding authors

from Sixteenth Annual Computational Neuroscience Meeting: CNS*2007

Toronto, Canada. 7-12 July 2007

Published: 6 July 2007

BMC Neuroscience 2007, 8(Suppl 2):PI30 doi:I0.I I86/I47|-2202-8-S2-PI30

(C) 2007 Hsi-Ping et al; licensee BioMed Central Ltd.

Thalamic and cortical V1 layer 4 neurons are capable of firing highly reliably and precisely upon repeated presentations of the same visual stimulus to the retina. To compare candidate causal mechanisms of spike-time reliability, a reconstructed multicompartment spiny stellate cell model with dynamic stochastic synapses was given varying synaptic inputs. We found reliability was primarily influenced by the number of synapses that fired synchronously during events (synchrony magnitude), which exhibits a supralinear relation; rather than by the rate of synchronous firing events (event rate) or synaptic strength, which exhibits comparatively more linear relations, even in the absence of voltage dependent conductances. Supralinear reliability highlights the efficacy of synchronous but weak synapses in driving output spiking, and may have implications for neural synchronicity within and between cortical areas. 